\title{
Metafronteira de produção e eficiência técnica da carcinicultura nos estados do Ceará e Rio Grande do Norte
}

\author{
Metafrontier production and technical efficiency of \\ shrimp farming in the States of Ceará and \\ Rio Grande do Norte
}

\author{
Jonathas Viana Monteiro ${ }^{1}$ (D), Alana Kedylla Monteiro Norões ${ }^{1}$ (D), \\ Rogério César Pereira de Araújo ${ }^{1}$ (D), Jair Andrade Araújo ${ }^{1}$ (D) e \\ Felipe Pinto da Silva ${ }^{2}$
}

\begin{abstract}
Resumo: No Nordeste do Brasil, a carcinicultura tem alcançado nível elevado de eficiência técnica, porém, estes avanços não vêm acontecendo de forma homogênea entre os estados produtores. Este estudo tem como objetivo analisar as diferenças tecnológicas da produção de camarão cultivado existentes entre os dois maiores produtores nacionais deste produto, Ceará e Rio Grande do Norte, em 2011. Para isto, aplicou-se o modelo de metafronteira tecnológica proposto por Battese, Rao e O'Donnell, que objetiva estimar uma função envoltória das fronteiras estocásticas de produção dos dois estados. Os dados relativos à produção e ao gerenciamento das fazendas de camarão foram extraídos do Censo da Carcinicultura de 2011, realizado pela Associação Brasileira de Criadores de Camarão (ABCC), sendo 100 fazendas localizadas no Ceará e 112, no Rio Grande do Norte. Os resultados mostraram que, em termos médios, o Ceará apresentou maior eficiência técnica (ET) do que o Rio Grande do Norte, sendo que a área média dos viveiros teve maior impacto sobre a ET do Ceará, e o número de funcionários contratados, sobre a ET do Rio Grande do Norte.
\end{abstract}

Palavras-chaves: camarão, eficiência produtiva, agronegócio.

Abstract: In the Northeast of Brazil, the shrimp farming has achieved high level of technical efficiency; however, these advances have not happened homogenously among producing states. This study aims to analyze the technological differences of shrimp production occurring among the two major producer states of this commodity, Ceará and Rio Grande do Norte, 2011. In order to perform this,

Data de submissão: 4 de maio de 2017. Data de aceite: 28 de outubro de 2018.

1. Universidade Federal do Ceará. Fortaleza, Ceará, Brasil. E-mail: jonathas0801@hotmail.com; alanadylla@hotmail.com; rcpa@ufc.br; jaraujoce@gmail.com

2. Universidade Estadual de Campinas, Campinas, São Paulo, Brasil. E-mail: dasilva.felipe0@gmail.com 
we applied the technological meta-frontier model proposed by Battese, Rao and O'Donnell, which aims to estimate an envelope function of the production stochastic frontier of the two states. Production and management data were extracted from the 2011 Shrimp Farming Census, conducted by the Brazilian Association of Shrimp Farming (ABCC), constituted of 100 farms in Ceará and 112 in Rio Grande do Norte. The results showed that, in average terms, Ceará was more technically efficient (ET) than Rio Grande do Norte, and the average area of ponds had higher impact over Ceará's ET, while the number of hired workers had higher impact over Rio Grande do Norte's ET.

Key-words: shrimp, productive efficiency, agribusiness.

Classificação JEL: D24, Q10.

\section{Introdução}

O cultivo de camarão marinho no mundo tem se expandido a uma taxa de $10 \%$ ao ano, constituindo-se em uma importante atividade econômica para os países em desenvolvimento. Em 2014, o Brasil produziu 65,1 mil toneladas de camarão marinho cultivado (Food and Agriculture Organization of United Nations, 2016), cujo destino tem sido os mercados doméstico e de exportação. Nesse mesmo ano, estima-se que a carcinicultura tenha gerado mais de 30 mil empregos diretos e indiretos.

A produção de camarão cultivado no Brasil está concentrada na região Nordeste, sendo os maiores produtores os estados do Ceará e Rio Grande do Norte. Em 2011, a região Nordeste sediava 92\% (ou 1.199) das fazendas, que eram responsáveis por 99,3\% (ou 69.171 t) da produção total no Brasil (Brasil, 2013). Em 2014, o Ceará, na primeira posição, produziu $58,3 \%$ do total, seguido pelo Rio Grande do Norte, com 25,5\% (Brasil, 2013; Instituto Brasileiro de Geografia e Estatística, 2015). Os principais fatores que contribuíram para o sucesso dessa atividade na região Nordeste foram sua extensa faixa litorânea, as condições edafoclimáticas e a topografia favorável ao cultivo da espécie de camarão Litopenaeus vannamei (Castro \& Pagani, 2004; Lisboa Filho et al., 2006).

A carcinicultura brasileira apresenta alta concentração fundiária, segundo dados do Censo Setorial do Camarão 2011. Considerando cinco categorias de produtores (micro, pequeno, médio ou grande), os micros e pequenos produtores (com área de até 10 hectares) representavam
$73,9 \%$ das fazendas de camarão e ocupavam apenas 13,5\% (ou 2.655 hectares) da área total das propriedades, enquanto os grandes produtores (com área maior que 50 hectares) correspondiam a apenas 6,3\% das propriedades e ocupavam $59,4 \%$ do total da área das propriedades (Brasil, 2013). A estrutura fundiária concentrada na carcinicultura pode ser um fator relevante em diferenciar os produtores quando a sua capacidade mobilizar recursos produtivos e adoção de tecnologias.

Na região Nordeste, os sistemas de produção semi-intensiva e intensiva têm predominado na carcinicultura. Por exemplo, segundo a ABCC, no Ceará, em 2011, 70\% das fazendas adotavam o sistema semi-intensivo de produção (Brasil, 2013). Esses dois níveis tecnológicos se diferenciam em função da densidade de estocagem dos viveiros (e.g. acima de 50 camarões $/ \mathrm{m}^{2}$ para intensivo), tipo de ração utilizada (desbalanceada ou balanceada) e forma de manejo e monitoramento da água (Araújo; Araújo, 2015). Já Silva \& Sampaio (2009) identificam que a carcinicultura praticada no Rio Grande do Norte caracteriza-se pelo predomínio de pequenas e médias fazendas, possuidoras de sistemas extensivo e semi-intensivo de produção.

Na década de 2000, na busca por aumentar o desempenho técnico dos cultivos, novas tecnologias foram desenvolvidas, tais como técnicas de reprodução das pós-larvas, formação de plantéis, uso de berçários intensivos, preparação e manejo dos viveiros, uso de ração adequada, comedouros fixos e monitoramento dos parâmetros de qualidade da água (Joventino, 2006). A adoção dessas tecnologias depende da capacidade 
do produtor de ter acesso ao crédito rural, o que tem sido apontado como uma barreira para a expansão da carcinicultura na região Nordeste (Kubitza, 2015).

Além do clima severo tipicamente encontrado na região Nordeste, uma estrutura fundiária concentrada, nível tecnológico dual (semi-intensivo e intensivo) e a diversidade de práticas de manejo da produção adotada pelas fazendas podem afetar o grau de eficiência técnica e econômica das regióes produtoras.

No Brasil, os estudos sobre a eficiência técnica da carcinicultura ainda são escassos. Oliveira (2008), através da análise envoltória de dados (DEA), avaliou a eficiência do cultivo do camarão marinho no Rio Grande do Norte. Sousa Júnior (2003) analisou a eficiência da produção de camarão marinho em cativeiro no estado do Ceará․․ Oliveira (2008), através da análise envoltória de dados (DEA), avaliou a eficiência do cultivo do camarão marinho no Rio Grande do Norte. Sousa Júnior (2003) analisou a eficiência da produção de camarão marinho em cativeiro no estado do Ceará. Silva \& Sampaio (2009) analisaram a eficiência técnica de pequenos e médios produtores de camarão no Rio Grande do Norte por meio da estimação das fronteiras de produção não paramétricas (DEA e FDH).

Em geral, esses estudos tinham como objetivo estimar a função de produção da carcinicultura - utilizando as abordagens paramétricas (e.g. fronteira estocástica) e não paramétricas (e.g. análise envoltória de dados) e identificar os principais fatores que causam ineficiência técnica em um determinado estado produtor (e.g. Ceará ou Rio Grande do Norte). Essas abordagens não permitem fazer comparações entre estados pelo fato de a fronteira de possibilidades de produção ser determinada endogenamente para cada estado. Nesse sentido, quando se compara a carcinicultura praticada no Ceará com a do Rio Grande do Norte, uma peculiaridade evidencia-se nesta última, como apresenta Silva \& Sampaio (2009). Segundo os autores, há um grande número de pequenos e médios produtores, que se caracterizam pela utilização de sistemas extensivos e semi-intensivo de produção, diferente do quadro encontrado no Ceará, caracterizado por produtores que utilizam sistemas semi-intensivo e intensivo de produção. Essas características regionais não

1 No mundo, a eficiência técnica da carcinicultura foi investigada na Malásia (Islam et al., 2014), Vietnã (Kiet \& Fisher, 2014), Bangladesh (Begum et al., 2013), e Índia (Sivaraman et al., 2015). devem ser negligenciadas e, para contornar esta limitação, nesse estudo, utiliza-se a função de metafronteira de produção, a qual permite comparar o grau de eficiência técnica entre regiões, tendo como base a mesma linha de referência (benchmark).

Desta forma, este estudo teve como objetivo principal analisar as diferenças tecnológicas da produção de camarão entre os dois maiores produtores nacionais, Ceará e Rio Grande do Norte. Para tanto, utilizou-se o método proposto por Battese et al. (2004), depois aprimorado por O'Donnell et al. (2008), para a estimação de uma metafronteira de produção. Este método consiste em, primeiramente, estimar as fronteiras de produção estocástica para cada região (estado) com base nos fatores de eficiência e ineficiência. Em seguida, uma função de metafronteira (envelope das funções de produção estocástica) é estimada para representar a eficiência técnica de referência que servirá de base para se comparar as eficiências técnicas de cada região.

O presente estudo está divido em quatro seções, além desta seção introdutória. Na segunda, apresentam-se o método de metafronteira e os estudos de análise de eficiência estocástica realizados no Brasil. Na terceira seção, foram mostrados os dados, as variáveis e o modelo empírico do estudo. Na quarta foram apresentados os resultados e discussão da análise de metafronteira estocástica. Por fim, na quinta seção, foram expostas as principais conclusões do estudo.

\section{Revisão de literatura}

\subsection{Análise de eficiência técnica}

Os métodos de mensuração de eficiência podem ser classificados em paramétricos e não paramétricos. Os métodos não paramétricos se diferenciam dos paramétricos pelo fato de o primeiro não exigir a especificação da função de produção ou custos, a mesma sendo estimada por meio de programação linear. Entre os métodos não paramétricos, destaca-se a análise de envoltória de dados (DEA). Ao contrário, os métodos paramétricos requerem a especificação de uma função de produção ou custos, a qual é estimada por meio de métodos econométricos. 
O método paramétrico da análise de fronteira estocástica, proposto por Aigner et al. (1977) e Meeusen \& Broeck (1977), tem se popularizado como ferramenta para medir a eficiência técnica de produção (Islam et al., 2014). Na abordagem de fronteira de produção, a eficiência técnica é definida, em termos de produção, como a quantidade máxima de produção física obtida a partir de uma dada combinação de insumos necessários (Farrel, 1957). Desta forma, a eficiência técnica é estimada por meio da razão entre a quantidade de produto observado e a quantidade máxima de produto possível, mantendo-se constante a quantidade de insumos, tecnologia e condições ambientais.

A análise da fronteira estocástica evoluiu para a análise de metafronteira estocástica, tendo como base o conceito de função de metaprodução cunhada por Hayami (1969) e Hayami \& Ruttan (1970). A função de metaprodução é um envelope das funções de produção tradicionais, assumindo que todos os produtores de diferentes grupos (países, regiões, entre outros) potencialmente têm acesso à mesma tecnologia. A abordagem de Metafronteira Estocástica (MFE) foi desenvolvida por Battese et al. (2004), tomando como pressuposto o fato que a tecnologia pode diferir entre regiões. Conceitualmente, a estimação da metafronteira representa o envoltório de todas as Fronteiras Estocásticas de Produção (FEP) de todos os grupos ou regiões, podendo sua delimitação ser definida em função de características geográficas, ambientais ou econômicas.

\subsection{Modelo de metafronteira estocástica}

Parte-se do pressuposto de que as firmas estão distribuídas em grupos, cada grupo compartilhando características semelhantes (e.g., região, atividade produtiva etc.). Para cada um desses grupos, é possível estimar uma fronteira estocástica de produção (FEP), como proposto por Meeusen \& Broeck (1977). Desta forma, o modelo geral da fronteira estocástica pode ser expresso por:

$Y_{i}^{j}=f\left(X_{i}^{j}, \beta^{j}\right) e^{\varepsilon_{i}^{j}}$

em que: $y_{i}{ }^{j}$ é o produto da unidade produtiva i, da j-ésima região; $x_{i}{ }^{j}$ é o vetor de insumos; e $\varepsilon_{i}{ }^{j}=v_{i}{ }^{j}-u_{i}{ }^{j}$ é o erro aleatório. Assumindo que o exponencial da fronteira de produção é linear no vetor de parâmetros $\beta^{j}$, a tecnologia pode ser representada por uma forma funcional adequada (i.e., Cobb-Douglas ou Translog).
Considera-se que a relação entre insumos e produtos das diversas firmas em cada grupo é representada por uma fronteira estocástica. Sendo assim, para cada j-ésimo grupo, existe uma amostra de $N_{j}$ firmas, produzindo um produto a partir de um conjunto de insumos; a fronteira estocástica para estes grupos é definida por:

$Y_{i}^{j}=f\left(X_{i}^{j}, \beta^{j}\right) \cdot e^{\left(v_{i}^{j}-u_{i}^{j}\right)}$

em que: $Y_{i}$ é a quantidade de produto observada na firma $i ; X_{i}$ é o vetor $1 x \mathrm{~K}$ de insumos ou outras variáveis explicativas, associadas com firma $i$; $\beta^{j}$ é o vetor $K x 1$ de parâmetros desconhecidos associados ao grupo $j ; v_{i}^{j}$ é o erro aleatório, independentes e identicamente distribuídos (iid), com distribuição normal de média zero e variância constante $\left(v_{i}^{j} \sim\right.$ iid $\left.N\left(0, \sigma_{v}^{2}\right)\right)$; e $u_{i}$ é um erro aleatório não negativo não observável associado à ineficiência técnica da firma $i$ do grupo $j$.

A ineficiência técnica da firma é entendida como um desvio da produção para baixo, tomando como referência a fronteira de possibilidade de produção. O erro aleatório $u_{i}$ pode assumir vários tipos de distribuição, por exemplo, a half-normal (HN) (Aigner et al., 1977), a normal truncada (Battese \& Coelli, 1995), a exponencial e a gama. As estimações desses parâmetros serão obtidas pelo método da máxima verossimilhança, partindo da hipótese de o termo da ineficiência possuir distribuição $\mathrm{HN}$, em que $u_{i} \sim$ iid $H N\left(0, \sigma_{v}^{2}\right)$, como proposto por Battese \& Coelli (1992). Segundo Battese \& Coelli (1992), a utilização da distribuição half-normal sustenta-se pelo fato de seu fácil trato em âmbito computacional e por apresentar robustez na estimação da eficiência técnica das firmas.

A estimação dos parâmetros da equação (2) é feita pelo método da máxima verossimilhança. Como mostrado em Battese \& Coelli (1992), o indicador de eficiência técnica para a firma i do grupo jé dado pela razão entre o produto observado e o produto da fronteira, expresso por:

$E T_{i}^{j}=\frac{y_{i}^{j}}{f\left(X_{i}^{j} \beta_{i}^{j}\right) \cdot e^{v_{i}^{j}}}=e^{-u_{i}^{j}}$

Depois de estimadas as FEPs individuais, faz-se necessário verificar se os vários grupos compartilham a mesma tecnologia. Isto pode ser feito por meio do teste da Razão de Verossimilhança (LR), em que $\mathrm{L}\left(\mathrm{H}_{0}\right)$ é o valor da função do log-verossimilhança para a fronteira estocástica estimada reunindo os dados de todos os grupos, e $\mathrm{L}\left(\mathrm{H}_{1}\right)$ é a soma dos valores das funções do log-verossimilhança das FEPs individuais. Os graus 
de liberdade para a estatística Chi-quadrado são a diferença entre o número dos parâmetros estimados sob $\mathrm{H}_{1}$ e $\mathrm{H}_{0}$. Se a hipótese $\mathrm{H}_{0}$ que define a fronteira estocástica para os dados conjuntos for rejeitada em favor das fronteiras individuais $\left(\mathrm{H}_{1}\right)$, então os dados não devem ser reunidos e, neste caso, a MFE é a abordagem apropriada para estimar e comparar a ET entre grupos ou regiões (Battese et al., 2004).

O modelo MFE é definido por Battese et al. (2004) como uma fronteira paramétrica determinística de forma funcional específica (e.g., Cobb-Douglas ou Translog) tal que o valor previsto da MFE seja maior ou igual ao valor estimado para a fronteira estocástica de qualquer uma das firmas ou grupos. O modelo MFE determinístico para todas as firmas que compõem os grupos pode ser expresso da seguinte forma:

$Y_{i}^{*}=f\left(x_{1 i}, x_{2 i}, \ldots, x_{K i} ; \beta^{*}\right)=e^{\left(x_{i}^{\prime} \beta^{*}\right)}$

em que: $Y_{i}^{*}$ e $\beta^{*}$ denotam o produto e o vetor de parâmetros do modelo MFE, respectivamente, desde que a seguinte condição se mantenha para todos os grupos $j(j=1,2, \ldots, J)$ :

$$
x_{i}^{\prime} \beta^{*} \geq x_{i}^{\prime} \beta^{j}
$$

A restrição, definida pela equação (5), garante que nenhuma fronteira regional seja maior que a metafronteira. O'Donnell et al. (2008) apresentaram duas formas para o cálculo $\beta^{*}$. O primeiro método é estimar uma metafronteira estocástica, usando os produtos, insumos de todas as firmas, independentemente da região e em todos os períodos, calculando uma metafronteira estocástica. Este método não garante que a metafronteira estimada seja a envoltória das fronteiras regionais, dada a possibilidade de haver problema de má especificação. O segundo método, proposto por Battese et al. (2004) e O'Donnell et al. (2008) considera que, dados os parâmetros estimados $\hat{\beta}^{j}$ das fronteiras regionais, a estimação da MF se dá na estimação de $\beta^{*}$ pela solução do seguinte problema de programação linear:

$$
\begin{aligned}
& \min _{\beta^{*}} \sum_{i=1}^{N}\left|\ln f\left(X_{i} ; \beta^{*}\right)-\ln f\left(X_{i} ; \hat{\beta}^{j}\right)\right| \\
& \text { s.a.: } \ln f\left(X_{i} ; \beta^{*}\right) \geq \ln f\left(X_{i} ; \hat{\beta}^{j}\right)
\end{aligned}
$$

Já que $\hat{\beta}^{j}$, o vetor de coeficientes estimados para a fronteira estocástica para cada grupo j e o vetor de insumos são considerados fixos, a forma equivalente do problema de PL da equação (6) pode ser especificada se a função $f\left(X_{i} ; \beta^{*}\right)$ for log-linear em seus parâmetros: $\min _{\beta^{*}} \bar{x} \beta^{*}$

$$
\text { s.a.: } x_{i}^{\prime} \beta^{*} \geq x_{i}^{\prime} \beta^{j}
$$

Com o problema de programação linear solucionado e os vetores $\beta^{*}$ da metafronteira e $\beta^{j}$ das fronteiras estocásticas individuais, o produto observado da firma $i$ do grupo $j$ pode ser expresso por:

$y_{i}^{j}=e^{\left(-u_{i}^{j}\right)} \times \frac{f\left(X_{i}^{j}, \beta^{j}\right)}{f\left(X_{i}^{j}, \beta^{*}\right)} \times f\left(X_{i}^{j}, \beta^{*}\right) e^{\left(v_{i}^{j}\right)}$

O lado direito da fórmula é formado por três componentes. O primeiro termo é igual ao da equação (3) e representa a eficiência técnica da firma $i$ relativa à fronteira estocástica do j-ésimo grupo $\left(E T_{i}^{j}\right)$. O segundo termo é denominado de Razão de Metatecnologia (RMT), que representa a diferença entre a tecnologia disponível para o grupo $j$ relativo à tecnologia disponível para todas as firmas e grupos, como mostra a equação (9).

$R M T_{i}^{j}=\frac{f\left(X_{i}^{j}, \beta^{j}\right)}{f\left(X_{i}^{j}, \beta^{*}\right)}$, tal que $\quad 0 \leq R M T_{i}^{j} \leq 1$

A RMT é obtida pela razão entre o produto da fronteira do grupo ${ }^{j}$ e o produto potencial da função de metafronteira, dados os insumos observados (Battese et al., 2004). A RMT identifica a razão do produto da função de fronteira de produção para cada grupo relativo ao produto potencial, que é definido pela função metafronteira, dados os insumos observados. A definição da RMT indica que o "aumento na razão de metatecnologia implica em decréscimo na diferença entre a fronteira do grupo e a metafronteira" (O'Donnell et al., 2008, p. 236). A RMT assume valor entre 0 e 1 , sendo que 1 indica nenhuma diferença entre a firma em um grupo e a metafronteira.

O terceiro termo da equação (8) indica a eficiência técnica da firma ${ }^{i}$ com relação à função de metafronteira, podendo ser expresso pela seguinte equação:

$E T_{i}^{* j}=\frac{y_{i}^{j}}{f\left(X_{i}^{j}, \beta^{*}\right) e^{\left(v_{i}^{j}\right)}}=E T_{i}^{j} \times R M T_{i}^{j}$

\subsection{Eficiência técnica da carcinicultura}

Estudos que aplicaram o método de análise de fronteira estocástica para avaliar a eficiência técnica da carcinicultura brasileira são limitados. Nos parágrafos 
seguintes serão apresentadas pesquisas realizadas no âmbito da análise de eficiência técnica no Brasil e da aplicação do método de metafronteira na carcinicultura.

Sousa Júnior (2003) fez uso de um dos métodos possíveis para estimar a fronteira de produção, em 2002: a Análise de Envoltória de Dados (DEA). Analisou a eficiência técnica da produção de camarão marinho em cativeiro no estado do Ceará. Para analisar quantos dos 68 carcinicultores amostrados eram eficientes, foi selecionada uma gama de variáveis importantes na produção, tais como: densidade de estocagem, aeração artificial, espécie utilizada, oxigênio dissolvido, número de viveiros, área de viveiros, dentre outras. O escore de eficiência técnica foi utilizado para verificar se as firmas eram ou não eficientes, com esse valor variando de 0 a 1, e a firma estaria na fronteira de produção se o escore estive-se acima de 0,9 . Foi observado que, dos 68 produtores, 38 se mostraram tecnicamente eficientes, representando $56 \%$ dos estabelecimentos estudados. Observou-se também que os produtores classificados como eficientes adotavam o sistema de produção semi-intensivo, que admite uma densidade de estocagem dos viveiros de 30 a $60 \mathrm{cam} / \mathrm{m}^{2}$.

No Rio Grande do Norte, no período compreendido entre 2002 e 2005, Oliveira (2008) avaliou a eficiência do cultivo de camarão marinho em sistema dulciaquícola. Para a análise de eficiência técnica, o autor utilizou a Análise de Envoltória de Dados (DEA) multiproduto e trabalhou com um banco de dados composto por 115 fazendas. Foram utilizadas algumas variáveis para verificar a eficiência do cultivo de camarão marinho, são elas: produção e peso médio final, como variáveis de produto; tempo de cultivo, quantidade de pós-larva, área do viveiro, quantidade de ração ofertada, marca da ração e laboratório de origem das pós-larvas, indicando as variáveis de insumo. Com base nos resultados das 115 fazendas amostradas, verificou-se que somente 24 se mostraram tecnicamente eficientes e 91, ineficientes, considerando que, para ser eficiente, o escore da fazenda deveria estar acima de 0,9 .

Oliveira (2008) ainda observou que as variáveis temperatura e peso final dos camarões apresentaram relação direta com a produção, pois o aumento dessas variáveis gerava ganhos de produção para o carcinicultor a um nível de significância de 5\%. Já as variáveis oxigênio, $\mathrm{pH}$ e transparência não foram significativas a um nível de $5 \%$. Desta forma, concluiu-se que aproximadamente $80 \%$ das fazendas de carcinicultura do estado do Rio
Grande do Norte foram tecnicamente ineficientes entre 2002 e 2005.

Em 2009, Silva \& Sampaio (2009) realizaram, no estado do Rio Grande do Norte, uma análise de eficiência técnica para dois sistemas de produção de camarão marinho (extensivo e semi-intensivo) por meio da estimação da fronteira de produção não paramétrica, a partir de uma amostra de 348 pequenos e médios produtores de camarão, com área inferior a 50 ha. Foi observado que, para os sistemas de produção extensivo e semi-intensivo, os níveis de eficiência técnica foram mais altos. Isso foi explicado pelo fato de algumas práticas de gestão estarem associadas diretamente aos escores de eficiência, como: o uso de aeradores, equipamentos para monitoração da água, tratamento de solo, utilização de tanques berçários entre outros. Porém, algumas variáveis de gestão não foram significativas a um nível de 5\%, sendo elas: a presença de bandejas para ração, que pode ser explicada pela falta de conhecimento do nível adequado do balanceamento da ração que deve ser empregado nas bandejas.

Silva \& Sampaio (2009) concluíram que produtores localizados em estuários com menor nível de emissões de nitrogênio e fósforo obtiveram maior escore de eficiência técnica, e a colocação das rações por meio do sistema de bandejas reduzia o desperdício e ajudava a diminuir a formação de sedimentos no fundo dos viveiros, melhorando a qualidade dos camarões e, consequentemente, aumentando a eficiência técnica das fazendas, pois o elevado nível de resíduos no substrato pode provocar a mortalidade de camarões.

No contexto mundial, merece destaque o trabalho de Kiet \& Fisher (2014). Os autores investigaram a eficiência das práticas de cultivo de camarão intensiva, semi-intensiva e extensiva, bem como a diferença entre a eficiência de fazendas de camarão localizadas a montante e a jusante do delta do rio Mekong, Vietnã. A eficiência das fazendas de camarão foi medida usando a análise de metafronteira em uma amostra de 292 fazendas. Os resultados indicaram que os carcinicultores estavam, em média, a operar de forma ineficiente. A eficiência técnica estimada das fazendas de camarão em relação à fronteira de grupo nas duas regiões foram de $70 \%$, $53 \%$ e $31,3 \%$ para fazendas semi-intensivas, intensivas e extensivas, o que significa que, em média, os carcinicultores dos cultivos semi-intensivos, intensivos e extensivos poderiam melhorar sua produtividade por meio da redução de 30\%, 47\% e 68,7\% dos insumos sem reduzir a produção, respectivamente. 


\section{Metodologia}

\subsection{Fonte de dados}

A análise da função de metafronteira estocástica utilizada neste estudo tem como foco as fazendas de carcinicultura de portes micro, pequeno, médio e grande, especializadas na engorda de camarão, localizadas no Ceará e no Rio Grande do Norte. Esses estados foram selecionados para a análise por serem os dois maiores produtores nacionais de camarão marinho, sendo responsáveis, conjuntamente, por $83,8 \%$ da produção de camarão marinho nacional, em 2011.

Em 2011, o Ceará tinha 325 produtores de camarão que ocupavam área de 6.580 ha de viveiros em operação, com área média de 20,2 ha/fazenda, distribuídos em 21 municípios. Nesse mesmo ano, a produção de camarão foi de $31.982 \mathrm{t}$, tendo alcançado produtividade média de $4,86 \mathrm{t} / \mathrm{ha} / \mathrm{ano}$. Por sua vez, o Rio Grande do Norte tinha 361 unidades de produção, que ocupavam uma área em operação de 6.540 ha, distribuídos em 27 municípios, que indicam produtividade média de 2,73 t/ ha/ano e produção anual de $17.825 \mathrm{t}$.

Os dados utilizados na análise são de natureza secundária, extraídos do Censo Setorial do Camarão, realizado pela Associação Brasileira de Criadores de Camarão (Brasil, 2013), em 2011.

Do total de fazendas levantadas pelo censo no Ceará e no Rio Grande do Norte (686), foram utilizados na análise efetivamente $31 \%$ das observações, ou seja, 212 observações (100 para o CE e 112 para o $\mathrm{RN}$ ), tendo em vista a necessidade de eliminar observações com dados faltosos.

\subsection{Modelo empírico}

No presente estudo o método escolhido para a mensuração empírica da eficiência técnica e da razão de metatecnologia foi o proposto por Battese \& Coelli (1992) e O’Donnell et al. (2008).

A construção da metafronteira de produção se deu em dois estágios. O primeiro é realizado pela construção de fronteiras estocásticas regionais, utilizando o método proposto por Battese \& Coelli (1992). Já o segundo estágio consiste em estimar a metafronteira por técnicas de programação linear.
Primeiro, seguindo o método proposto por Battese \& Coelli (1992), são estimadas duas funções de fronteira estocástica, uma para cada grupo de produtores, as quais foram definidas em função de sua localização geográfica: (1) produtores do estado do Ceará e (2) produtores do estado do Rio Grande do Norte.

Uma forma funcional única foi escolhida para estimar a função de fronteira estocástica de produção do Ceará e Rio Grande do Norte. A fronteira de produção estocástica é especificada da seguinte forma:

$\ln \operatorname{Prod}_{i}^{j}=\beta_{0}+\beta_{1} \ln N F u n c_{i}^{j}+\beta_{2} \ln N v i v_{i}^{j}+\beta_{3} \ln A v i v_{i}^{j}+v_{i}-u_{i}$

em que: $\operatorname{Prod}_{i}^{j}$ é a quantidade de camarão produzida na fazenda $i$ no estado j; $N F u n c_{i}^{j}$ é o número total de funcionários contratados da fazenda $i$ no estado $j ; N v i v_{i}^{j}$ é o número de viveiros em operação da fazenda $i$ no estado j; $A v i v_{i}^{j}$ é a área dos viveiros em hectares da fazenda $i$ no estado j; $v_{i}$ é erro aleatório da função de produção que por hipótese uma distribuição normal iid $\sim N\left(0, \sigma^{2}\right)$; e $u_{i}$ é erro aleatório associado à ineficiência técnica da produção que por hipótese assume uma distribuição Half-Normal iid $\sim H N\left(0, \sigma^{2}\right)$. Todas as variáveis estão em logaritmo neperiano (ln).

A estimação dos parâmetros da equação (11) é feita pelo método da máxima verossimilhança, o que permite o cálculo das magnitudes das eficiências técnicas para cada uma das fazendas de carcinicultura da amostra e, consequentemente, possibilitando a estimação das fronteiras regionais (Ceará e Rio Grande do Norte), quanto à fronteira agrupada.

A estimação dos parâmetros da metafronteira tecnológica se deu por meio da otimização do problema de programação linear apresentado na metodologia, utilizando o software estatístico Shazam.

\subsection{Testes realizados}

\subsubsection{Efeito da ineficiência técnica na função de produção}

Testa-se a inexistência da ineficiência técnica, ou seja, se de fato o modelo capta a ineficiência da firma. Neste caso, toma-se o valor da log-verossimilhança (LL) do modelo estimado sem esta variável. Pelo método de mínimos quadrados ordinários, aplica-se o teste da razão de verossimilhança generalizada (LR), comparando-se ao 
valor crítico da tabela de Kodde \& Palm (1986). Os graus de liberdade são correspondentes às variáveis definidas pela função de produção, definida anteriormente. Então, tem-se:

$H_{0}$ : Modelo de mínimos quadrados ordinários (inexistência de ineficiência técnica, $\gamma=0$ ).

$H_{1}$ : A ineficiência técnica deve ser considerada no modelo, $\gamma \neq 0$.

$L R=-2\left[\ln L L H_{0}-L n L L H_{1}\right] \sim X^{2}$.

LR > T KP (Tabela de Kodde \& Palm, 1986); rejeita-se $H_{0}$.

\subsubsection{Existência de duas fronteiras regionais}

Para testar a hipótese de que os dois estados podem ser representados pela mesma fronteira de produção estocástica, utiliza-se o teste da razão de verossimilhança generalizada. Então, tem-se:

$H_{0}$ : Os estados do Ceará e do Rio Grande do Norte possuem a mesma fronteira de possibilidades de produção e produzem segundo tecnologias semelhantes. Não há necessidade de estimar duas fronteiras distintas.

$H_{1}$ : Os estados utilizam tecnologias diferentes e possuem fronteiras de possibilidades de produção distintas.

$L R=-2\left[\ln L L H_{0}-L n L L H_{1}\right] \sim X^{2}$

LR > T KP (Tabela de Kodde \& Palm, 1986); rejeita-se $H_{0}$.
Se a hipótese nula for rejeitada, a fronteira de produção agrupada é rejeitada e aceita-se a hipótese alternativa de existência de fronteiras regionais distintas.

\section{Resultados e discussão}

Nesta seção são apresentadas as estatístcas descritivas das variáveis utilizadas, bem como os resultados da estimação das fronteiras regionais e os testes de existência de ineficiência técnica e de existência de fronteiras regionais. Em seguida, mostra-se o modelo estimado de metafronteira de produção.

\subsection{Estatísticas descritivas}

A Tabela 1 apresenta a definição das variáveis que representam o produto e os insumos empregados na produção, incluídas no modelo de metafronteira estocástica e suas estatísticas descritivas. Os dados de produção abrangem os ciclos produtivos realizados durante 2011.

A produção de camarão (ProdC), medida em toneladas, é a variável dependente e representa o desempenho produtivo da fazenda durante o período de análise (Sousa Júnior, 2003; Silva \& Sampaio, 2009). As variáveis independentes são o número total de funcionários contratados (NFunc), o número de viveiros em operação (Nviv) e a área média dos viveiros (Aviv).

Tabela 1. Estatística descritiva das variáveis utilizadas na estimação da metafronteira de produção da carcinicultura

\begin{tabular}{|c|c|c|c|c|c|c|c|}
\hline Variáveis & Definição & Estado $^{1}$ & Média & $\begin{array}{l}\text { Desvio } \\
\text { Padrão }\end{array}$ & Min. & Max. & $\mathrm{CV}^{2}$ \\
\hline \multicolumn{8}{|l|}{ Dependente } \\
\hline \multirow{2}{*}{ ProdC } & \multirow{2}{*}{ Produção de camarão (toneladas) } & CE & 200,3 & 444,5 & 4,0 & $4.150,0$ & 221,9 \\
\hline & & $\mathrm{RN}$ & 70,8 & 125,4 & 1,8 & 700,0 & 177,1 \\
\hline \multicolumn{8}{|c|}{ Independentes } \\
\hline \multirow{2}{*}{ NFunc } & \multirow{2}{*}{ Número de funcionários contratados } & $\mathrm{CE}$ & 16,4 & 35,2 & 1,0 & 320,0 & 214,7 \\
\hline & & $\mathrm{RN}$ & 6,8 & 11,7 & 1,0 & 93,0 & 172,1 \\
\hline \multirow{2}{*}{ Nviv } & \multirow{2}{*}{ Número de viveiros em operação } & $\mathrm{CE}$ & 11,9 & 23,4 & 0,5 & 220,0 & 196,4 \\
\hline & & $\mathrm{RN}$ & 7,8 & 8,9 & 0,5 & 52,0 & 113,8 \\
\hline \multirow{2}{*}{ Aviv } & \multirow{2}{*}{ Área média dos viveiros (ha) } & $\mathrm{CE}$ & 2,5 & 1,7 & 1,0 & 12,0 & 67,6 \\
\hline & & $\mathrm{RN}$ & 3,3 & 1,7 & 7,9 & 80,7 & 51,3 \\
\hline \multirow{2}{*}{\multicolumn{2}{|c|}{ Total de observações }} & CE & & & 100 & & \\
\hline & & RN & & & 112 & & \\
\hline
\end{tabular}

Fonte: elaboração dos autores a partir dos dados da pesquisa.

Nota: (1) CE é o estado do Ceará e RN é o estado do Rio Grande do Norte; (2) CV é o coeficiente de variação, calculado da seguinte forma: $C V=\frac{\sigma}{\bar{X}} x 100$. 
A média da produção de camarão por fazenda apresentou-se consideravelmente maior para os produtores do Ceará (CE), com produção média de 200,3 toneladas e desvio padrão de 444,5 toneladas, enquanto o Rio Grande do Norte (RN) tem produção média de 70,8 toneladas, com desvio padrão de 125,4 toneladas, estando esta produção mais dispersa dentre os produtores do Ceará $(\mathrm{CV}=221,9 \%)$ do que os do Rio Grande do Norte $(\mathrm{CV}=177,1 \%)$ (Tabela 1). A produção de camarão no CE variou no intervalo de 4 a $4.150 \mathrm{t}$, enquanto a produção do RN variou entre 1,8 e $700 \mathrm{t}$ por fazenda.

A quantidade de mão de obra (NFunc) expressa a força do trabalho empregada nessa atividade. O número médio de funcionários foi maior no estado do CE (Média = 16,4) do que no RN (Média =6,9), sendo o Ceará o estado com maior coeficiente de variação $(\mathrm{CV}=214,7 \%)$. Isto significa que as fazendas do $\mathrm{CE}$, quando comparadas às do RN, empregam maior quantidade de mão de obra por fazenda, embora observe-se elevada variância entre as fazendas.
O número e área dos viveiros em operação conjuntamente expressam a escala de produção nas fazendas. Comparando os estados do CE e RN, verifica-se que as fazendas do Ceará utilizavam, em média, maior número de viveiros (Média $=11,9$ ), com menor área média de lâmina d'água (Média = 2,5), enquanto as fazendas do Rio Grande do Norte produziam com menor número de viveiros (Média =7,8), mas com maior área de lâmina d'água (Média = 3,3). Portanto, existe uma tendência de os produtores cearenses conduzirem sua produção de camarão com um sistema mais intensivo do que os produtores potiguares, o que pode ser uma explicação para a maior produção observada no Ceará.

\subsection{Análise das fronteiras estocásticas}

Na Tabela 2 são apresentadas as estimativas dos três modelos de fronteira estocástica: (i) Agrupada, em que se reuniu todos os produtores em uma única base de dados; (ii) RN, apenas com os produtores do Rio Grande do Norte e (iii) CE, apenas com os produtores do Ceará.

Tabela 2. Fronteiras estocásticas estimadas

\begin{tabular}{|c|c|c|c|c|c|c|}
\hline \multirow{2}{*}{ Variáveis } & \multicolumn{2}{|c|}{ AGRUPADA } & \multicolumn{2}{|c|}{ RN } & \multicolumn{2}{|c|}{$\mathrm{CE}$} \\
\hline & Coef. & $P>|z|$ & Coef. & $P>|z|$ & Coef. & $P>|z|$ \\
\hline$\beta_{0}($ Const. $)$ & 2,221 & 0,001 & 2,144 & 0,001 & 2,482 & 0,001 \\
\hline$\beta_{I}(\ln$ NFunc $)$ & 0,664 & 0,001 & 0,562 & 0,001 & 0,544 & 0,001 \\
\hline$\beta_{2}(\ln N v i v)$ & 0,422 & 0,001 & 0,516 & 0,001 & 0,417 & 0,016 \\
\hline$\beta_{3}(\ln A v i v)$ & 0,423 & 0,001 & 0,294 & 0,001 & 0,693 & 0,001 \\
\hline$\sigma^{2}$ & 0,545 & 0,008 & 0,456 & 0,054 & 0,659 & 0,018 \\
\hline$\gamma$ & 0,701 & 0,002 & 0,729 & 0,004 & 0,860 & 0,001 \\
\hline$\sigma_{u}^{2}$ & 0,382 & 0,009 & 0,333 & 0,025 & 0,567 & 0,001 \\
\hline$\sigma_{v}^{2}$ & 0,163 & 0,001 & 0,124 & 0,008 & 0,092 & 0,031 \\
\hline$\sigma$ & 0,739 & 0,001 & 0,675 & 0,001 & 0,812 & 0,001 \\
\hline$\sigma_{u}$ & 0,618 & 0,002 & 0,577 & 0,017 & 0,753 & 0,001 \\
\hline$\sigma_{v}$ & 0,404 & 0,001 & 0,352 & 0,003 & 0,304 & 0,010 \\
\hline$\lambda$ & 1,532 & 0,002 & 1,640 & 0,010 & 2,479 & 0,005 \\
\hline Log-verossimilhança & \multicolumn{2}{|c|}{$-172,105$} & \multicolumn{2}{|c|}{$-78,878$} & \multicolumn{2}{|c|}{$-78,472$} \\
\hline ET Média & \multicolumn{2}{|c|}{0,650} & \multicolumn{2}{|c|}{0,668} & \multicolumn{2}{|c|}{0,602} \\
\hline Obs. & \multicolumn{2}{|c|}{212} & \multicolumn{2}{|c|}{112} & \multicolumn{2}{|c|}{100} \\
\hline
\end{tabular}

Fonte: elaboração dos autores a partir dos dados da pesquisa. 
Nos três modelos, todos os coeficientes estimados foram significativos a um nível de significância de 5\% e com os sinais esperados. Isto indica que a função Cobb-Douglas ajustou satisfatoriamente os dados e que os insumos considerados nos modelos como variáveis explicativas foram relevantes em explicar a variabilidade da produção em ambos os estados.

Vale ressaltar, que a estimação da fronteira agrupada é fundamental para a realização do teste da existência ou não de diferenças regionais, como apresentado na metodologia, que embase a existência de fronteiras de produção distintas entre a carcinicultura praticada no Ceará e no Rio Grande do Norte, e que motiva o uso da metafronteira de produção.

Todos os fatores de produção incluídos na função da fronteira estocástica estão associados positivamente à produção média da fazenda em ambas as fronteiras regionais, embora a magnitude de seus impactos varie entre regiões.

Em termos de número total de funcionários contratados (NFunc), o coeficiente desta variável no modelo CE $($ Coef. $=0,544)$ foi ligeiramente menor do que o observado no modelo RN (Coef. =0,562). Isto significa que o impacto da mão de obra sobre a produção, tanto no CE quanto no RN, é inelástico, ou seja, o aumento de $1 \%$ no número de funcionários nas fazendas no Ceará aumentaria a produção em $0,544 \%$ para os produtores cearenses, já para o Rio Grande do Norte, uma variação de $1 \%$ no número de funcionários resultaria em uma elevação de $0,562 \%$ na produção. Isso aponta para o fato de que as fazendas nesses estados já podem estar operando na região eficiente de produção e sob o efeito dos rendimentos decrescentes do fator, já que o aumento do número de funcionários implica num aumento menos que proporcional na sua produção.

Em termos de mão de obra, os incrementos da produção podem se originar a partir do aumento da qualidade da mão de obra, já que este tem sido um fator apontado como limitante para a expansão da carcinicultura no Nordeste (Kubitza, 2015). Isto seria alcançado por meio de investimento em capacitação e treinamento no setor. Estudando a carcinicultura na Malásia, Islam et al. (2014) observaram que, quanto maior o nível educacional e a experiência dos funcionários, menor é a ineficiência das fazendas.

O impacto do número de viveiros sobre a produção variou, em magnitude, entre os estados. O coeficiente do
Nviv do modelo CE (Coef. = 0,417) mostrou-se menor do que o observado no modelo RN (Coef. =0,516). Para os produtores do $\mathrm{RN}$, seu coeficiente revela que o aumento de $1 \%$ no número de viveiros em operação implica no acréscimo de $0,516 \%$ na produção de camarão deste estado. Portanto, os produtores do estado do Rio Grande do Norte poderiam experimentar aumento de eficiência na produção de camarão aumentando o número de viveiros em sua fazenda.

Foi observado por Sousa Júnior (2003) que o número médio de viveiros em operação no Ceará em 2002 era de cinco viveiros/firma, e o aumento no número de viveiros não gerava impactos significativos na produção de camarão. Entretanto, neste estudo, foi visto que, em 2011, o número médio de viveiros, tanto para o estado do Ceará (11,9 viveiros / firma) como para o Rio Grande do Norte (7,8 viveiros / firma), foi bem acima daquele verificado por Sousa Júnior (2003). Porém, mesmo com esse aumento, o impacto desta variável na produção ainda continuou sendo pouco expressivo, mas, comparando-se às variáveis analisadas para o Rio Grande do Norte, era uma das mais importantes para a produção. Para tanto, o autor utilizou uma amostra de 68 produtores, em 2002, nos principais estuários do estado do Ceará (Remédios, Coreaú, Acaraú, Zumbi, Curu, Choró, Pirangi e Jaguaribe).

Quanto ao insumo área média de viveiros (Aviv), os impactos desse fator sobre a eficiência da produção nos dois estados foram marcantemente diferentes. Este fator foi o que causou maior impacto na produção das fazendas no estado do Ceará (Coef. = 0,693), enquanto no estado do Rio Grande do Norte, foi o que causou menor impacto (Coef. $=0,294$ ). Dessa forma, verifica-se uma diferença de $0,4 \%$ na magnitude do efeito deste fator sobre a produção entre os estados.

Oliveira (2008), utilizando dados de 115 cultivos, oriundos de 22 viveiros de uma fazendo no estado do Rio Grande do Norte, em 2008, verificou que se fazia uso de uma área média de viveiros de 3,35 ha, e que o aumento deste insumo não gerava ganhos expressivos na produção das fazendas potiguares. O mesmo padrão foi observado para 2011, verificado nesta pesquisa, pois a área média dos viveiros permaneceu praticamente a mesma, de 3,3 ha, e a influência desta variável, comparada às outras, contida no modelo de fronteira produção estocástica, foi também de pouca magnitude. 
Já em relação ao estado do Ceará, Sousa Júnior (2003), em 2002, verificou que os carcinicultores utilizavam, em média, 19,25 ha de área de viveiro, sendo que o aumento desse insumo não gerava ganhos significativos na produção. Entretanto, nesta pesquisa, foi visto que, em 2011, as fazendas do Ceará tinham, em média, apenas 2,5 ha de área de viveiro, redução de mais de 16 ha, e o aumento dessa variável implicava a maior influência para gerar ganhos de produção para as firmas, comparando-se às outras variáveis desse modelo de fronteira estocástica. Isto pode ser explicado devido ao aprimoramento do pacote tecnológico e ao ganho de experiência dos carcinicultores, obtidos com o passar desses nove anos de desenvolvimento da carcinicultura, podendo aumentar a produção, que foi de $60.128 \mathrm{t}$ em 2002 , para $65.671 \mathrm{t}$ em 2011, além do fato de ser tecnicamente eficiente fazendo uso de uma menor área de viveiros (Food and Agriculture Organization of United Nations, 2014).

Os fatores que causam maior impacto sobre a produção de camarão divergiram entre os estados analisados. Segundo o modelo RN, na ordem decrescente, os fatores de produção de maior impacto foram NFunc, Nviv e Aviv. Por sua vez, de acordo com o modelo CE, os fatores de produção de maior impacto foram Aviv, NFunc e Nviv. Pelo modelo AGRUPADA, o fator de maior impacto na produção foi NFunc (Coef. $=0,664$ ), seguidos do Nviv e Aviv, os quais apresentaram, aproximadamente, o mesmo efeito marginal (Coef. $=0,42$ ).

Do ponto de vista de gerenciamento do setor, os incentivos visando o aumento da eficiência da carcinicultura variam em função do recorte geográfico. Enquanto o estado do Ceará teria maiores ganhos de produção por meio da expansão da área dos viveiros, o estado do Rio Grande do Norte teria ganhos mais elevados por meio do aumento do emprego da mão de obra nas fazendas. De forma geral, considerando os dois estados, os maiores ganhos de eficiência da produção de camarão seriam alcançados por meio da contratação de mão de obra, o que também proporcionaria benefícios socioeconômicos para a região. Segundo Nascimento (2013), a carcinicultura representa uma atividade econômica importante, pois contribui para a fixação do homem no Nordeste brasileiro e melhora a distribuição geográfica da renda no País.

O indicador de ineficiência técnica, $\gamma\left(=\sigma_{v}^{2} / \sigma^{2}\right)$ foi estimado em mais de 0,7 para os dois estados do Nordeste em estudo. Isto significa que mais de $70 \%$ da variância total do erro composto dos modelos correspondem à variância da ineficiência técnica. Com este resultado é possível afirmar que há uma evidência forte da presença de ineficiência técnica nas firmas produtoras de camarão cultivado, tanto para o Rio Grande do Norte como para o Ceará.

A média da eficiência técnica da produção de camarão (ET Média) variou entre as fronteiras regionais. O Ceará apresentou ET média de 60\%, e o Rio Grande do Norte obteve o escore médio de eficiência de $67 \%$. Isto significa que os produtores cearenses empregam $60 \%$ do nível tecnológico disponível para os produtores deste estado. Em relação aos produtores potiguares, o percentual de adoção corresponde a $67 \%$ do nível tecnológico disponível a todos os produtores do Rio Grande do Norte.

Esta diferença não implica que a carcinicultura cearense apresente nível de eficiência técnica inferior àquela observada na carcinicultura potiguar, uma vez que a comparação das duas fronteiras somente faria sentido se a tecnologia de produção utilizada pelos produtores dos dois estados não fosse a mesma; para tanto, testes de hipóteses foram realizados e apresentados na próxima seção. Porém, se as evidências apresentadas nos teste de hipóteses mostrarem que os dois estados utilizam práticas originadas do mesmo pacote tecnológico, a comparação direta das duas fronteiras regionais é inadequada e a análise deve ser feita com base no modelo de dados agrupados.

\subsection{Análise dos testes de hipóteses}

Após a estimação dos modelos de fronteira estocástica regionais, foram realizados dois testes: (i) teste de ineficiência técnica e o teste de existência de fronteiras regionais. A Tabela 3 apresenta os resultados.

Quanto ao teste de ausência de ineficiência técnica, a hipótese nula $\left(H_{0}\right)$ define que o modelo não capta o efeito de ineficiência, sendo expresso por $\gamma=0$. Caso o teste falhe em rejeitar $H_{0}$, deve-se utilizar o método de Mínimos Quadrados Ordinários (MQO) para estimar o modelo.

O teste de inexistência de ineficiência foi aplicado aos três modelos estimados de fronteiras estocástica: AGRUPADA, CE e RN. Os resultados mostraram que a hipótese nula foi rejeitada nos três modelos, ou seja, os valores estimados $(\lambda)$ superam seus valores críticos ao nível de $5 \%$ de significância na tabela de Kodde \& Palm (1986), dados seus graus de liberdade. Portanto, 
rejeita-se a hipótese nula que estabelece a inexistência da ineficiência técnica no modelo determinado, o que demonstra a existência de ineficiência na produção de camarão e justifica a estimativa do modelo de eficiência estocástica.

Por fim, foi realizado o teste para validar a existência da diferenciação entre as possibilidades produtivas entre os dois estados ou não. Nesse teste, a hipótese nula $\left(H_{0}\right)$ define que a tecnologia de produção empregada pelos estados não se diferencia significativamente. Conforme os resultados, a hipótese nula foi rejeitada e aceita a hipótese alternativa, a qual declara que os estados do Ceará e Rio Grande do Norte empregam diferentes tecnologias de produção na carcinicultura. Logo, pode-se utilizar o quadro teórico da metafronteira de produção, a fim de estimar e comparar as eficiências técnicas de produção da carcinicultura dos dois estados em estudo.

\subsection{Análise das eficiências técnicas e razão de metafronteira}

O modelo de metafronteira estocástica (modelo MFE) estimou parâmetros para os fatores de produção iguais àqueles estimados pelo modelo de fronteira estocástica dos carcinicultores do estado do Ceará (modelo CE). Este resultado indica que a tecnologia de produção empregada na carcinicultura cearense permitiu alcançar nível de eficiência técnica maior do que aquela obtida pela carcinicultura potiguar. Desta forma, o padrão tecnológico utilizado pela carcinicultura do Ceará é a fronteira tecnológica para todos os carcinicultores que operavam nos dois estados, sendo aí onde se situam as melhores práticas disponíveis para o setor.

Na Tabela 4 são mostradas as estimativas de eficiências e de razão de metatecnologia (RMT). Os carcinicultores do estado do Ceará se situaram na metafronteira de produção. Desta forma, a razão de metatecnologia, definida na equação (9), é igual a 1 para todos os produtores cearenses. A consequência desta afirmação está no fato de que o Ceará exaure as possibilidades tecnológicas existentes para os dois estados. Consequentemente, como se pode observar na Tabela 4, a RMT aponta o quanto a fronteira do Rio Grande do Norte está abaixo da região definidas como metafronteira (a metadistância).

Os carcinicultores do estado do Rio Grande do Norte apresentaram RMT média de 0,68 e desvio padrão de 0,08 . Esse resultado indica que, em média, a produção dos carcinicultores potiguares foi, em 2011, 68\% do máximo que os carcinicultores cearenses podiam produzir naquele ano, ao utilizarem os mesmos fatores de produção e tecnologias disponíveis no estado do Ceará.

A ET referente à metafronteira de produção é chamada de $\mathrm{ET}^{*}$ e foi estimada para todos os carcinicultores do Rio Grande do Norte $\left(\mathrm{ET}_{\mathrm{RN}}^{*}\right)$, de acordo com a equação (10). A ET* dos carcinicultores do Ceará $\left(E^{*} T_{C E}\right)$ é igual à ET dos carcinicultores estimada pela fronteira regional desse estado $\left(\mathrm{ET}_{\mathrm{CE}}\right)$, por serem os produtores desse estado a própria metafronteira de produção.

Tabela 3. Testes de razão verossimilhança dos parâmetros das fronteiras de produção

\begin{tabular}{llccccc}
\hline \multicolumn{1}{c}{ Testes } & Fronteira & Hipótese nula & $\begin{array}{c}\text { Grau } \\
\text { de liberdade }\end{array}$ & Valor de $\lambda$ & Valor crítico & Decisão (5\%) \\
\hline \multirow{2}{*}{$\begin{array}{lllcll}\text { Inexistência de ineficiência } \\
\text { técnica }\end{array}$} & AGRUPADA & $H_{0}: \gamma=0$ & 1 & 3,793 & 2,706 & Rejeita $H_{0}$ \\
& CE & $H_{0}: \gamma=0$ & 1 & 6,832 & 2,706 & Rejeita $H_{0}$ \\
$\begin{array}{l}\text { Existência de duas } \\
\text { fronteiras regionais }\end{array}$ & Não se aplica & $\begin{array}{c}H_{0}: \text { Fronteira } \\
\text { agrupada }\end{array}$ & 6 & 2,881 & 2,706 & Rejeita $H_{0}$ \\
\hline
\end{tabular}

Fonte: elaboração dos autores a partir dos dados da pesquisa.

Tabela 4. Estatística descritiva das ETs e RMTs estimadas

\begin{tabular}{|c|c|c|c|c|}
\hline Estatísticas & $\mathrm{ET}_{\mathrm{RN}}$ & $\mathrm{RMT}_{\mathrm{RN}}$ & $\mathrm{ET}_{\mathrm{RN}}^{*}$ & $\mathrm{ET}_{\mathrm{CE}}=\mathrm{ET}_{\mathrm{CE}}^{*}$ \\
\hline Média & 0,67 & 0,68 & 0,46 & 0,60 \\
\hline Desvio padrão & 0,14 & 0,08 & 0,11 & 0,18 \\
\hline Mínimo & 0,21 & 0,38 & 0,16 & 0,15 \\
\hline Máximo & 0,89 & 0,89 & 0,66 & 0,90 \\
\hline
\end{tabular}

Fonte: elaboração dos autores a partir dos dados da pesquisa. 
$\mathrm{A} \mathrm{ET}_{\mathrm{RN}}^{*}$ foi estimada em $46 \%$, tendo o carcinicultor com menor $\mathrm{ET}^{*}$ neste estado, obtido escore de $16 \%$ e aquele com maior $\mathrm{ET}^{*}, 66 \%$. A média do $\mathrm{ET}_{\mathrm{CE}}^{*}$ foi $60 \%$, mostrando-se consideravelmente superior à do estado do Rio Grande do Norte. Os escores do ET* dos carcinicultores do estado do Ceará variaram entre $15 \%$ e $90 \%$.

A Tabela 5 apresenta as distribuições de frequências das ET* dos carcinicultores do Ceará e Rio Grande do Norte. Os carcinicultores potiguares obtiveram escores de eficiência técnica de metafronteira de até 0,7. A faixa de eficiência com maior frequência foi $0,4-0,5$, com 36,6\% das fazendas (ou 41 fazendas). A maioria das fazendas (64,3\% ou 72 fazendas) ficou no intervalo de eficiência entre 0,4 e 0,6 .

Já os carcinicultura cearenses obtiveram escores de eficiência técnica de metafronteira de até 0,9 , não tendo sido observadas fazendas com eficiência na faixa 0,9-1,0. A maior frequência de eficiência está localizada na faixa entre 0,5 e 0,9 , em que estão situadas $73 \%$ das fazendas.

A Tabela 6 mostra a ET* e as variáveis explicativas dos cinco carcinicultores mais eficientes e menos eficientes dos estados do Ceará e Rio Grande do Norte.

Tabela 5. Distribuição de frequência absoluta e relativa das ETs e RMTs estimadas

\begin{tabular}{|c|c|c|c|c|c|c|c|c|}
\hline \multirow{2}{*}{ Intervalo } & \multicolumn{2}{|c|}{$\mathrm{ET}_{\mathrm{RN}}$} & \multicolumn{2}{|c|}{$\mathrm{RMT}_{\mathrm{RN}}$} & \multicolumn{2}{|c|}{$\mathrm{ET}^{*}{ }_{\mathrm{RN}}$} & \multicolumn{2}{|c|}{$\mathrm{ET}_{\mathrm{CE}}=\mathrm{ET}_{\mathrm{CE}}^{*}$} \\
\hline & N. & $\%$ & N. & $\%$ & N. & $\%$ & N. & $\%$ \\
\hline $0,1-0,2$ & 0 & 0,0 & 0 & 0,0 & 2 & 1,8 & 3 & 3 \\
\hline $0,2-0,3$ & 2 & 1,8 & 0 & 0,0 & 9 & 8,0 & 3 & 3 \\
\hline $0,3-0,4$ & 2 & 1,8 & 1 & 0,9 & 20 & 17,9 & 12 & 12 \\
\hline $0,4-0,5$ & 12 & 10,7 & 2 & 1,8 & 41 & 36,6 & 9 & 9 \\
\hline $0,5-0,6$ & 15 & 13,4 & 9 & 8,0 & 31 & 27,7 & 16 & 16 \\
\hline $0,6-0,7$ & 25 & 22,3 & 52 & 46,4 & 9 & 8,0 & 21 & 21 \\
\hline $0,7-0,8$ & 38 & 33,9 & 44 & 39,3 & 0 & 0,0 & 21 & 21 \\
\hline $0,8-0,9$ & 18 & 16,1 & 4 & 3,6 & 0 & 0,0 & 15 & 15 \\
\hline $0,9-1,0$ & 0 & 0,0 & 0 & 0,0 & 0 & 0,0 & 0 & 0 \\
\hline Total & 112 & 100 & 112 & 100 & 112 & 100 & 100 & 100 \\
\hline
\end{tabular}

Fonte: elaboração dos autores a partir dos dados da pesquisa.

Tabela 6. Comparação dos cinco produtores mais eficientes com os cinco menos eficientes dos estados do Ceará e Rio Grande do Norte

\begin{tabular}{|c|c|c|c|c|c|c|c|}
\hline Ranking & Regiões & Produtor & $\mathbf{E T}^{*}$ & ProdC & NFunc & Nviv & Aviv \\
\hline \multirow{2}{*}{$1^{\mathrm{o}}$} & CE & 25 & 0,90 & 210 & 8 & 5 & 2 \\
\hline & $\mathrm{RN}$ & 94 & 0,66 & 28 & 1 & 4 & 1 \\
\hline \multirow{2}{*}{$2^{\mathrm{o}}$} & CE & 20 & 0,88 & 150 & 9 & 0,5 & 6 \\
\hline & $\mathrm{RN}$ & 43 & 0,65 & 660 & 28 & 52 & 2 \\
\hline \multirow{2}{*}{$3^{o}$} & $\mathrm{CE}$ & 11 & 0,87 & 45 & 2 & 4 & 1 \\
\hline & $\mathrm{RN}$ & 22 & 0,64 & 40 & 3 & 8 & 0,81 \\
\hline \multirow{2}{*}{$4^{\circ}$} & $\mathrm{CE}$ & 81 & 0,87 & 280 & 10 & 17 & 1,7 \\
\hline & $\mathrm{RN}$ & 67 & 0,62 & 20 & 1 & 4 & 1 \\
\hline \multirow{2}{*}{$5^{\mathrm{o}}$} & $\mathrm{CE}$ & 75 & 0,84 & 168 & 11 & 12 & 1,2 \\
\hline & $\mathrm{RN}$ & 87 & 0,62 & 130 & 10 & 7 & 1,57 \\
\hline $96^{\circ}$ & $\mathrm{CE}$ & 27 & 0,23 & 30 & 6 & 5 & 4,5 \\
\hline $108^{\circ}$ & $\mathrm{RN}$ & 47 & 0,26 & 11,5 & 3 & 3 & 3,2 \\
\hline $97^{\circ}$ & CE & 64 & 0,22 & 13,5 & 4 & 5 & 2 \\
\hline $109^{\circ}$ & $\mathrm{RN}$ & 36 & 0,24 & 15 & 6 & 10 & 1,2 \\
\hline $98^{\circ}$ & $\mathrm{CE}$ & 86 & 0,16 & 6 & 4 & 3 & 1,5 \\
\hline $110^{\circ}$ & $\mathrm{RN}$ & 79 & 0,20 & 26 & 9 & 13 & 2,6 \\
\hline $99^{\circ}$ & $\mathrm{CE}$ & 90 & 0,16 & 8,4 & 5 & 2 & 2,7 \\
\hline $111^{\circ}$ & $\mathrm{RN}$ & 107 & 0,20 & 9,2 & 1 & 2 & 20 \\
\hline $100^{\circ}$ & $\mathrm{CE}$ & 48 & 0,15 & 4 & 3 & 4 & 1 \\
\hline $112^{\mathrm{o}}$ & $\mathrm{RN}$ & 84 & 0,16 & 1,8 & 2 & 2 & 0,75 \\
\hline
\end{tabular}

Fonte: elaboração dos autores a partir dos dados da pesquisa. 
Comparando os produtores mais eficientes dos dois estados, produtores 25 e 94, respectivamente, diferenciaram-se de forma marcante em termos de eficiência: o carcinicultor cearense possui $90 \%$; e o carcinicultor potiguar, $66 \%$. Em termos de produção de camarão (ProdC), o carcinicultor do CE produziu 7,5 vezes a mais do que o carcinicultor do RN. A quantidade de todos os insumos empregados na carcinicultura do produtor do CE foi maior do que os empregados pelo produtor do RN. A maior diferença foi observada para a quantidade de mão de obra contratada (NFunc). Isso pode ser uma explicação para a elevada produção do carcinicultor 25 em relação ao 94, corroborando com os resultados de Islam et al. (2014).

Analisando os cinco últimos produtores dos dois estados, observa-se que esses carcinicultores apresentaram escores de eficiência abaixo de 0,27. Também pode ser visto que os carcinicultores situados na parte inferior do ranking apresentaram escores muito próximos, em termos de eficiência técnica. Por exemplo, os últimos colocados para os dois estados apresentaram escores de 0,15 (CE) e 0,16 (RN), com diferença de apenas 0,1 entre os escores.

\section{Conclusões}

Este estudo se propôs a analisar a eficiência técnica da carcinicultura e suas diferenças entre os dois maiores produtores nacionais, Ceará e Rio Grande do Norte. Com a utilização do modelo de metafronteira estocástica proposto por Battese et al. (2004) e O'Donnell et al. (2008), foram estimadas as ET, ET* e RMT dos carcinicultores desses estados.

A partir dos procedimentos metodológicos utilizados na pesquisa, pode-se afirmar que a carcinicultura do Ceará e a do Rio Grande do Norte não compartilham a mesma tecnologia de produção. Em 2011, os carcinicultores do estado do Ceará possuíam a maior eficiência técnica média relativa à metafronteira, de $60 \%$, enquanto os carcinicultores do Rio Grande do Norte apresentaram eficiência técnica média de $46 \%$.

A razão de metatecnologia (RMT) estimada para os carcinicultores do Rio Grande do Norte indicou que, mesmo utilizando os mesmos fatores de produção e tecnologias disponíveis no estado do Ceará, os carcinicultores potiguares produziram $68 \%$ da produção máxima que pode ser obtida pelos carcinicultores cearenses.

Todas as variáveis escolhidas para a análise se mostraram significativas, porém, com diferença quanto à magnitude do impacto causado por essas variáveis em cada estado. Na carcinicultura cearense, a variável "área média dos viveiros" é a que causou maior impacto, apresentando coeficiente de 0,693. Já para a carcinicultura potiguar, o fator de maior impacto foi o número de funcionários contratados, com coeficiente de 0,562 .

\section{Referências}

Aigner, D. J., Lovell, C. A. K., \& Schmidt, P. (1977). Formulation and Estimation of Stochastic Frontier Production Function Models. Journal of Econometrics, 6, 21-37.

Araújo, A. M. M., \& Araújo, R. C. P. (2015, julho 26-29). Análise das práticas de gestão ambiental e seus impactos sobre a produtividade da carcinicultura no Ceará. In: Anais do 53ํㅡㅁ Congresso da Sociedade Brasileira de Economia, Administração e Sociologia Rural - Sober (19 p.) João Pessoa: Sociedade Brasileira de Economia, Administração e Sociologia Rural - Sober.

Battese, G. E., \& Coelli, T. J. (1992). Frontier production functions, technical efficiency and panel data: with application to paddy farmers in India. Journal of Productivity Analysis, 3, 153-169.

Battese, G. E., \& Coelli, T. J. (1995). A model for technical inefficiency effects in a stochastic frontier production function for panel data. Empirical Economics, 20(2), 325-332.

Battese, G. E., Rao, D. S. P., \& O'Donnell, C. J. (2004). A metafrontier production function for estimation of technical efficiencies and technology gaps for firms operating under different technologies. Journal of Productivity Analysis, 21, 91-103.

Begum, M. E. A., Hossain, M. I., \& Papnagioto, E. (2013). Technical efficiency of shrimp farming in Bangladesh: an application of the stochastic production frontier approach. Journal of the World Aquaculture Society, 44(5), 641-654.

Brasil. Ministério da Pesca e Agricultura. (2013). Levantamento da infraestrutura produtiva e dos aspectos tecnológicos, econômicos, sociais e ambientais da carcinicultura marinha no Brasil em 2011. Natal: ABCC. Recuperado em 03 de outubro de 2015, de http:/ / abccam.com.br/ wp-content/uploads/2013/12/LEVANTAMENTO-DAINFRAESTRUTURA-PRODUTIVA.pdf

Castro, A. A., \& Pagani, G. D. (2004). Secagem e composição química da cabeça de camarão (Litopenaeus vannamei 
Boone) a diferentes temperaturas. Revista Brasileira de Produtos Agroindustriais, 6(2), 123-129.

Farrel, M. J. (1957). The measurement of production efficiency. Journal of the Royal Statistical Society, Series A, 120(3), 253-290.

Food and Agriculture Organization of United Nations - FAO. (2014). Seção fisheries and aquaculture department. Roma: FAO.

Food and Agriculture Organization of United Nations - FAO. (2016). The state of world fisheries and aquaculture: contributing to food security and nutrition for all. Roma: FAO.

Hayami, Y. (1969). Sources of agricultural productivity gap among selected countries. American Journal of Agricultural Economics, 51(3), 564-575.

Hayami, Y., \& Ruttan, V. W. (1970). Agricultural productivity differences among countries. American Economic Review, 60(5), 895-911.

Instituto Brasileiro de Geografia e Estatística - IBGE. (2015). Produção da pecuária municipal (v. 43, p. 1-49). Rio de Janeiro: IBGE.

Islam, G. M. N., Yew, T. S., \& Noh, K. M. (2014). Technical efficiency analysis of shrimp farming in Peninsular Malaysia: a stochastic frontier production function approach. Trends in Applied Sciences Research, 9(2), 103.

Joventino, F. K. P. (2006). A sustentabilidade da carcinicultura no município de Fortim-CE, com ênfase nos aspectos sociais, ambientais e tecnológicos. (Dissertação de mestrado). Programa de Pós-graduação em desenvolvimento e Meio Ambiente da Universidade Federal do Ceará. 114 p. Fortaleza.

Kiet, N. T., \& Fisher, T. C. G. (2014). Efficiency analysis and the effect of pollution on shrimp farms in the Mekong river delta. Aquaculture Economics \& Management, 18, 325-343.

Kodde, D. A., \& Palm, F. C. (1986). Wald criteria for jointly testing equality and inequality restrictions. Econometrica, 54(5), 1243-1248.
Kubitza, F. (2015). Aquicultura no Brasil: principais espécies, áreas de cultivo, rações, fatores limitantes e desafios. Panorama da Aquicultura, 25(150), jul./ago.

Lisboa Filho, W., Carlini Junior, R. J., Lucena, H. A., \& Silva, F. S. C. M. (2006). A Carcinicultura como uma viável e promissora alternativa econômica para a Região Nordeste. Pernambuco. Recuperado em 10 de dezembro 2016, de www.sober.org.br/palestra / 2/1096.pdf.

Meeusen, W., \& Broeck, J. V. D. Efficiency estimation from Cobb-Douglas production functions with composed error. International Economic Review, 18, 435-444, 1977.

Nascimento, M. L. (2013). Análise dos perigos associados ao camarão Litopenaeus vannamei no Brasil (Dissertação de mestrado). Faculdade de Medicina Veterinária e Zootecnia, Universidade de São Paulo, São Paulo. 86 p.

O’Donnell, C. J., Rao, D. S. P., \& Battese, G. E. (2008). Metafrontier frameworks for the study of firm-level efficiencies and technology ratios. Empirical Economics, 34(2), 231-255.

Oliveira, I. R. (2008). Utilização da análise envoltória de dados (DEA), no diagnóstico da eficiência de cultivo do camarão marinho Litopenaeus vannamei (BOONE, 1931) (Dissertação de mestrado). Universidade Federal de Pernambuco, Recife. 51 p.

Silva, J. L. M., \& Sampaio, L. M. B. (2009). Eficiência, gestão e meio ambiente na carcinicultura do Rio Grande do Norte. Revista de Economia e Sociologia Rural, 47(4), 883-902.

Sivaraman, I., Krishnan, M., Ananthan, P. S., Satyasai, K. J. S., Krishnan, L., Haribabu, P., \& Ananth, P. N. (2015). Technical efficiency of shrimp farming in Andhra Pradesh: estimation and implications. Current World Environment, Madhya Pradesh, 10(1), 199-205.

Sousa Júnior, J. P. (2003). Análise da eficiência da produção de camarão marinho em cativeiro no estado do Ceará. (Dissertação de mestrado). Universidade Federal do Ceará, Fortaleza. 142 p. 